\title{
Haplotype and phylogenetic analysis of OLR1(Intron I) gene in Jaffarabadi and Surti buffalo
}

\author{
Nadeem Shabir*1 ${ }^{1}$ C V J awale ${ }^{1}$, C D Bhong ${ }^{1}$, Mehrajuddin Naikoo $^{2}$, D N Rank ${ }^{3}$, C G J oshi $^{1}$ \\ Anand Veterinary College, \\ Anand Agricultural University, Anand - 388001, Gujarat, India. \\ 1. Department of Animal Biotechnology, 2. Department of Veterinary Gynecology and \\ Obstetrics, 3. Department of Animal Genetics and Breeding \\ * Corresponding author email : drnadurose@gmail.com
}

Received: 25-02-2011, Accepted: 14-03-2011, Published Online: 16-07-2011

doi: $10.5455 /$ vetworld.2011.396-398

\begin{abstract}
The present study was carried out to reveal haplotype and phylogenetic analysis of $O L R 1$ gene in Jaffarabadi and Surti breeds of buffalo. Twenty nucleotide sequences generated from our previous study (Shabir, 2009) were used to reveal the haplotypes in OLRI (Intron I) gene in the population of Jaffarabadi and Surti buffalo. Four haplotypes viz. H1 (CTA), H2 (TCT), H3 (CTT) and H4 (TTT) were observed with frequencies of 0.05, 0.1, 0.15 and 0.7 respectively in the population studied. Phylogenetic analysis of these twenty sequences with the same region of Bos taurus distributed the sequences into four clusters based on the homology between them. Cluster I contained two sequences ( jb3 and jb8) bearing a common SNP at nucleotide position 843. Cluster III contained s3, s4 and s7 since they possessed a common SNP at nucleotide position 423, however $\mathrm{s} 3$ remained as an out group in this cluster since it contained an additional SNP at position 866. The remaining sequences had highest homology and fell in cluster II while as the sequence of Bos taurus remained as an out group on account of the difference in bases at many loci.
\end{abstract}

Keywords: Phylogenetics, haplotypes, OLRI and Bubalus bubalis.

\section{Introduction}

Oxidized Low Density lipoprotein Receptor 1 $(O L R 1)$ is the major protein that binds, internalizes, and degrades oxidized low-density lipoprotein. The role of OLRI in lipid metabolism and the results of previous whole genome scan studies prompted the investigation of $O L R I$ as a candidate gene affecting milk composition traits (Khatib et. al., 2006). In another study conducted in Dutch Holstein-Friesian cattle population in $O L R 1$ gene to estimate genotype effects on milk production traits revealed that $O L R_{\mathrm{g} .8232 \mathrm{C}>\mathrm{A}}$ had a significant effect $(P<0.05)$ on milkfat percentage (Schennink et al., 2009).

The oxidized form of the low-density lipoprotein (oxLDL) is involved in endothelial cell injury, dysfunction, and activation, all of which are implicated in the development of atherosclerosis (Mehta and Li, 1998). It has been shown that oxLDL and its lipid constituents have numerous damaging effects on secretory activities of the endothelium, including induction of apoptosis (Imanishi et al., 2002). The major protein that binds, internalizes, and degrades oxLDL, oxidized LDL receptor 1 (OLRI), was initially identified in bovine aortic endothelial cells (Sawamura et al. (1997). In addition to binding oxLDL, OLRI removes aged and apoptotic cells from blood circulation (Oka et al., 1998). The bovine OLRI gene encodes $270 \mathrm{AA}$ and has $72 \%$ identity to the human protein (Sawamura et al., 1997). Aoyama et al. (1999) determined the structure of human OLR1, and found 6 exons, of which the first 3 corresponded to the $\mathrm{N}$-terminal cytoplasmic, transmembrane, and connecting neck domains, and the last 3 encoded the lectin domain. The genomic sequence of bovine $O L R 1$, recently released by Baylor College of Medicine, contains 5 exons \{GenBank accession no. NW_215807\}.

Based on the aforementioned studies indicating the role of $O L R I$ in lipid metabolism and its correlation with milk fat percentage, the study of haplotype analysis in this gene in Jaffarabadi and Surti breed of Bubalus bubalis was performed considering the higher milk fat percentages in buffaloes. Also, the phylogenetic analysis at OLRI gene was done to reveal the genetic distance between the two breeds. 


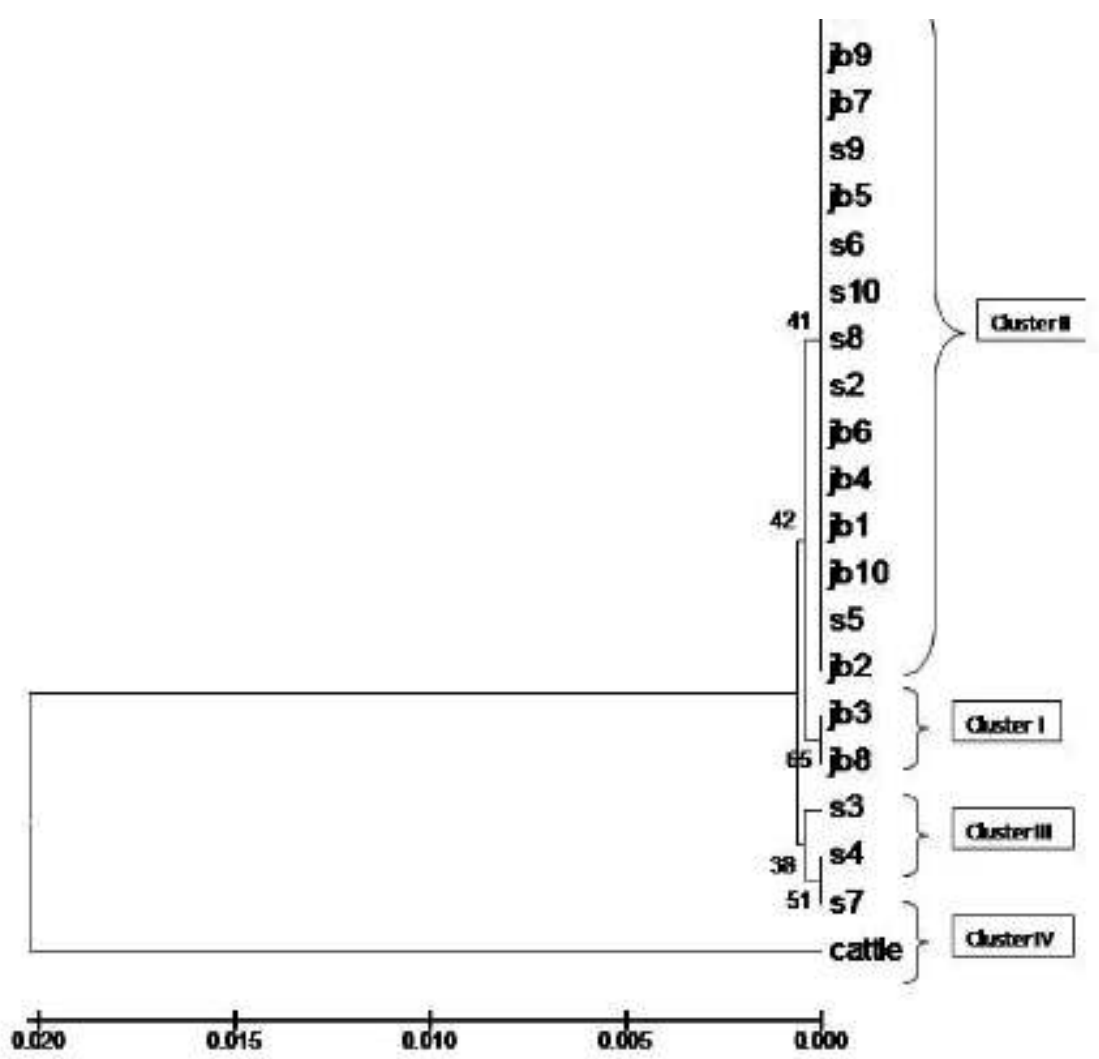

Figure-1. Phylogenetic placement of OLR 1 intron I DNA sequences of $J$ affarabadi and Surti breed of Bubalus bubalis and reference sequence of Bos taurus. The databank sequences have been marked by sample numbers. The number around the nodes are confidence levels $(\%)$ generated from 500 bootstrap trails. The scale bar is in fixed nucleotide substitutions per sequence position. $(\mathrm{J} b=\mathrm{J}$ afarabadi and $\mathrm{S}=\mathrm{Surti}$

\section{Materials and Methods}

Phylogenetic analysis: The nucleotide sequences of OLRI (Intron I) gene of Jaffarabadi and Surti buffalo published in National Centre for Biotechnology Information (NCBI) bearing accession numbers GQ478023 to GQ478042 (Shabir, 2009) were used to carry out the phylogenetic analysis of the twenty animals considered in our previous study. The phylogenetic analysis was carried out by MEGA4 software. The evolutionary history was inferred using the Unweighted Pair Group Method with Arithmetic Mean (UPGMA) method. The evolutionary distances were computed using the Maximum Composite Likelihood method in the units of the number of base substitutions per site. All positions containing gaps and missing data were eliminated from the dataset (Complete deletion option). The sample numbers jb1 to jb10 correspond to the accession numbers GQ478023 to GQ478032 while as that from s1 to s10 correspond to GQ478033 to GQ478042.
Haplotype Analysis: The SNPs detected in OLRI (Intron I) gene of Jaffarabadi and Surti buffalo in our previous study (Shabir, 2009) were used to carry out the haplotype analysis. The analysis was done by using the possible number of genotypic combinations of the Single Nucleotide Polymorphisms (SNPs) that could be formed.

\section{Results and Discussion}

\section{Phylogenetic placement of OLR1 gene}

The evolutionary history was inferred using the UPGMA method. The optimal tree with the sum of branch length of 0.04198671 is shown. The percentage of replicate trees in which the associated taxa clustered together in the bootstrap test (1000 replicates) as shown next to the branches in Fig.1. The tree was drawn to scale, with branch lengths in the same units as those of the evolutionary distances used to infer the phylogenetic tree. There were a total of 1147 positions in the final dataset. Phylogenetic analyses were conducted in MEGA4. On phylogenetic analysis of 
Tables-1. SNP positions and base changes in OLRI I ntron I in J affarabadi and Surti breed of Bubalus bubalis

\begin{tabular}{lcl}
\hline \multicolumn{3}{c}{ SNP Positions } \\
\hline 423 & 843 & 866 \\
$\mathrm{~T} / \mathrm{C}(\mathrm{A} / \mathrm{a})$ & $\mathrm{T} / \mathrm{C}(\mathrm{B} / \mathrm{b})$ & $\mathrm{T} / \mathrm{A}(\mathrm{C} / \mathrm{c})$ \\
\hline
\end{tabular}

twenty OLRI Intron I DNA sequences of Bubalus bubalis, the phylogentic tree formed four clusters, with each cluster containing highly similar sequences. Cluster I contained sequences Jb3 and Jb8, since both the sequences revealed an SNP at position 843 . Similarly, cluster III contained three sequences viz. S3, S4 and S7, since all the three sequences possessed a common SNP at position 423. However, S3 remained as an outgroup in cluster III since it possessed an additional SNP at position 866. The rest of the sequences were highly similar without any SNP and they fell in cluster II at $64 \%$ confidence level. The Cluster IV was the most different with least homology as it included the OLR 1 Intron I of Bos taurus. Since there were differences in the nucleotides in Bos taurus at many loci as compared to Bubalus bubalis, so on phylogenetic analysis it remained as an outgroup.

\section{Haplotype Analysis of the SNP detected}

Since three SNP (Table-1) were detected on multiple alignment of the twenty OLR 1 Intron I sequences in Bubalus bubalis in our previous study (Shabir, 2009), possible number of genotypic combinations that could be formed are $\left(2^{\mathrm{m}}=8\right)$ considering biallelic status of SNP. However, only 4 haplotypes were observed here as given in Table 2 . The frequency of haplotypes H1 (CTA), H2 (TCT), H3 (CTT) and H4 (TTT) in the population of 20 Jaffarabadi and Surti buffaloes was found to be 0.05 , $0.1,0.15$ and 0.7 respectively. In a similar study, Khatib et al. (2007) reported four intragenic haplotypes comprising SNP positions 7160, 7161, $7278,7381,7409,7438,7512$, and 8232 in $O L R 1$ gene of Holstein cattle and Haplotype [C; A; C] was associated with a significant increase in fat percentage when compared with the other haplotypes.
Table-2. Observed haplotypes and their frequencies.

\begin{tabular}{lllll}
\hline Haplotype & H1 & H2 & H3 & H4 \\
\hline Combinations & CTA & TCT & CTT & TTT \\
Number & 1 & 2 & 3 & 14 \\
Frequency & 0.05 & 0.1 & 0.15 & 0.70 \\
\hline
\end{tabular}

\section{Acknowledgement}

Authors are thankful to Indian Council for Agricultural Research (ICAR) for the financial help rendered by them.

\section{References}

1. Aoyama, T., et.al. (1999). Structure and chromosomal assignment of the human lectin-like oxidized low-densitylipoprotein receptor-1 (LOX-1) gene. Biochem J. 339:177-184.

2. Imanishi, T., Hano, T., Sawamura, T., Takarada, S. and Nishio I. (2002). Oxidized low density lipoprotein potentiation of Fas-induced apoptosis through lectin-like oxidized-low density lipoprotein receptor-1 in human umbilical vascular endothelial cells. Circ. J. 66: 1060-1064.

3. Khatib, H., et.al. (2006). Association of the OLR1 gene with milk composition in hostein dairy cattle. J. Dairy. Sci. 89: 1753-1760.

4. Khatib, H., Rosa, G. J. M., Weigel, K., Schiavini, F., Santus, E. and Bagnato, A. (2007). Additional support for an association between $O L R 1$ and milk fat traits in cattle. Ani. Genet. 38: 308-310

5. Mehta, J. L. and Li, D. Y. (1998). Identification and autoregulation of receptor for OX-LDL in cultured human coronary artery endothelial cells. Biochem. Biophys. Res. Commun. 248: 511-514.

6. Oka, K. T., et.al. (1998). Lectin-like oxidized low-density lipoprotein receptor 1 mediates phagocytosis of aged/apoptotic cells in endothelial cells. Proc Natl Acad Sci. 95:9535-9540.

7. Sawamura, T. N., Kume, T., Aoyama, H., Moriwaki, H., Hoshikawa, Y., Aiba, T., Tanaka, S., Miwa, Y., Katsura, T., Kita and Masaki, T. (1997). An endothelial receptor for oxidized low-density lipoprotein. Nature 386:73-77.

8. Schennink, A., Bovenhuis, H., Léon-Kloosterziel, K. M., van Arendonk, J. A. M., Visker, M. H. P. W. (2009) Effect of polymorphisms in the FASN, OLR1, PPARGC1A, PRL and STAT5A genes on bovine milk-fat composition. Ani Genet. 40:909-916.

9. Shabir, N. (2009). M.V.Sc. Thesis, Anand Agricultural University, Anand. 